Pacific Journal of Mathematics

SOLUTION FOR AN INTEGRAL EQUATION WITH 


\section{SOLUTION FOR AN INTEGRAL EQUATION WITH CONTINUOUS INTERVAL FUNCTIONS}

\section{J. A. ChatField}

Suppose $R$ is the set of real numbers and all integrals are of the subdivision-refinement type. Suppose each of $G$ and $H$ is a function from $R \times R$ to $R$ and each of $f$ and $h$ is a function from $R$ to $R$ such that $f(a)=h(a), d h$ is of bounded variation on $[a, x]$, and $\int_{a}^{x} H^{2}=\int_{a}^{x} G^{2}=0$ for $x>a$. The following two statements are equivalent:

(1) If $x>a$, then $f$ is bounded on $[a, x], \int_{a}^{x} H$ exists, $\int_{a}^{x} G$ exists, $(R L) \int_{a}^{x}(f G+f H)$ exists, and

$$
f(x)=h(x)+(R L) \int_{a}^{x}(f G+f H) ;
$$

(2) If $a \leqq p<q \leqq x$, then each of ${ }_{p} \Pi^{q}(1+H)$ and ${ }_{p} \Pi^{q}(1-G)^{-1}$ exists and neither is zero,

$$
(R) \int_{a}^{x}\left[{ }_{t} \Pi^{x}(1+H)(1+G)\right]\left[(1-G)^{-1}\right] d h
$$

exists, and

$$
\begin{aligned}
f(x)= & f(a)_{a} \Pi^{x}(1+H)(1-G)^{-1} \\
& \left.+(R) \int_{a}^{x}\left[{ }_{t} \Pi^{x}(1+H)(1+G)\right][1-G)^{-1}\right] d h .
\end{aligned}
$$

Introduction. In a recent paper [4], B. W. Helton solved the equation $f(x)=h(x)+(R L) \int_{a}^{x}(f G+f H)$ using product integration. All functions involved were required to be of bounded variation and the existence of various integrals was also required. In a subsequent paper [9], J. C. Helton was able to reduce the conditions placed on $h$ to being a quasicontinuous function although other conditions such as requiring $G$ and $H$ to be of bounded variation were maintained. In still another paper [7], J. C. Helton was able to reduce the restrictions placed on $G$ and $H$ to that of being product bounded but he also used other restrictions not used in [4] or [9] such as requiring $h$ to be a constant function and $G(r, s)=$ $-G(s, r)$, a condition not unlike that of being additive. In this paper we are concerned with obtaining a solution for the equation $f(x)=h(x)+(R L) \int_{a}^{x}(f G+f H)$ without requiring either $G$ or $H$ to be of bounded variation or that $G(r, s)=-G(s, r)$ or that $h$ be a constant function. Instead, our major restriction placed on $G$ and 
$H$ is that each be continuous (i.e., $\int_{a}^{x} G^{2}=\int_{a}^{x} H^{2}=0$ ) and in this respect, we note that we have shown in an earlier paper [3] with W. P. Davis that neither of the two statements, (1) $\int_{a}^{b} G^{2}=0$ and (2) $G$ is of bounded variation on $[a, b]$, is a consequence of the other. Also, some functions are either required to be product bounded or shown to be product bounded and we note that the set of function having bounded variation on an interval is a proper subset of the set of functions which are product bounded on the same interval.

The reader is also referred to recent studies of D. L. Lovelady [15], [16], J. S. MacNerney [17], J. W. Neuberger [18] and J. C. Helton [8] for related results and to put the present result in perspective. For examples of solutions of integral equations using product integrals and heretofore known results, the reader is referred to [4, page 319-322] and [2].

DeFinitions AND Notations. All functions will be functions from $R \times R$ to $R$ or $R$ to $R$ where $R$ is the set of real numbers. All integrals are of the subdivision-refinement type and we will use upper case $(G)$ for functions from $R \times R$ to $R$ and lower case $(g)$ for functions from $R$ to $R$. If $G$ is a function from $R \times R$ to $R$ then the statement that $G$ is (a) product bounded, (b) of bounded variation, (c) bounded on $[a, b]$ means there is a number $M$ and a subdivision $D$ of $[a, b]$ such that if $D^{\prime}=\left\{x_{i}\right\}_{i=1}^{n}$ is a refinement of $D$, then

(a) if $0<p \leqq q \leqq n,\left|\prod_{i=p}^{q} 1+G\left(x_{i-1}, x_{i}\right)\right|<M$.

(b) $\sum_{i=1}^{n}\left|G\left(x_{i-1}, x_{i}\right)\right|<M$.

(c) if $0<i \leqq n$, then $\left|G\left(x_{i-1}, x_{i}\right)\right|<M$, respectively.

The statement that the function $G$ from $R \times R$ to $R$ is (a) product integrable, (b) sum integrable on $[a, b]$ means there is a number $A$ such that if $\varepsilon>0$ then there is a subdivision $D$ of $[a, b]$ such that if $D^{\prime}=\left\{x_{i}\right\}_{i=0}^{n}$ is a refinement of $D$, then

(a) $\left|\prod_{i=1}^{n}\left[1+G\left(x_{i-1}, x_{i}\right)\right]-A\right|<\varepsilon$.

(b) $\left|\sum_{i=1}^{n} G\left(x_{i-1}, x_{i}\right)-A\right|<\varepsilon$, respectively.

If $h$ is a function from $R$ to $R$ then $d h$ denotes the function $G$ from $R \times R$ to $R$ such that for each $x<y, G(x, y)=h(y)-h(x)$. If $G$ is a function from $R \times R$ to $R$ and $G(x, y)$ exists, then $x$ is assumed to be less than $y$.

The following notations will be used to facilitate writing:

$$
\begin{aligned}
& \prod_{i=1}^{n}\left[1+G\left(x_{i-1}, x_{i}\right)\right]=\prod_{D}\left(1+G_{i}\right), \\
& \sum_{i=1}^{n} G\left(x_{i-1}, x_{i}\right)=\sum_{D} G_{i},
\end{aligned}
$$




$$
d h_{i}=h\left(x_{i}\right)-h\left(x_{i-1}\right)
$$

and

$$
f\left(x_{i}\right)=f_{i}
$$

where $D=\left\{x_{i}\right\}_{i=0}^{n}$ is a subdivision of some interval and $0<i \leqq n$. Further, left and right integrals are used extensively and the appropriate approximating term is indicated by $\approx$.

$$
\begin{aligned}
(L R) \int_{a}^{b}(f H+f G) & \approx f\left(x_{i-1}\right) G\left(x_{i-1}, x_{i}\right)+f\left(x_{i}\right) G\left(x_{i-1}, x_{i}\right) \\
(R) \int_{a}^{b} \prod_{t}^{b}(1+G) d f & \approx\left[\prod_{x_{i}}^{b}(1+G)\right]\left[f\left(x_{j}\right)-f\left(x_{i-1}\right)\right] \\
(R R) \int_{a}^{b}(f H+f G) & \approx f\left(x_{i}\right) G\left(x_{i-1}, x_{i}\right)+f\left(x_{i}\right) G\left(x_{i-1}, x_{i}\right) .
\end{aligned}
$$

Theorems. The following lemmas are needed in the proof of our main results.

Lemma 1.1. If $G$ is a function from $R \times R$ to $R,{ }_{a} \Pi^{b}(1+G)$ exists and is not zero, and $\int_{a}^{b} G$ exists, then $G$ is bounded on $[a, b]$ [12, Theorem 6].

LEMmA 1.2. If $\int_{a}^{b} G^{2}=0$, then the following statements are equivalent:

(1) ${ }_{a} \Pi^{b}(1+G)$ exists and is not zero.

(2) $\int_{a}^{b} G$ exists.

Furthermore, if either (1) or (2) is true, then $\ln _{a} \Pi^{b}(1+G)=$ $\int_{a}^{b} G[3$, Theorem 3].

LEMma 1.3. If $G$ is a function from $R \times R$ to $R$ such that $\int_{a}^{b} G^{2}=0$, then there is a subdivision $D$ of $[a, b]$ and a number $M$ such that if $D^{\prime}=\left\{x_{i}\right\}_{i=0}^{n}$ is a refinement of $D$ and $0<i \leqq n$, then $\left(1-G_{i}\right)^{-1}$ exists and $\left|\left(1-G_{i}\right)^{-1}\right|<M$.

Proof. This lemma follows directly from the fact that $\int_{a}^{b} G^{2}=0$.

Lemma 1.4. Suppose $G$ is a function from $R \times R$ to $R$ such that $|G|<1$ on $[a, b],{ }_{a} \Pi^{b}(1+G)$ exists and is not zero, and there is a subdivision $D$ of $[a, b]$ and a number $M$ such that if $D^{\prime}$ is a refinement of $D$ then $\left[\Pi_{D^{\prime}}\left(1+G_{i}\right)^{-1}\right]$ and $\left|\Pi_{D^{\prime}}\left(1+G_{i}\right)^{-1}\right|<M$. Then, there is a subdivision $D$ of $[a, b]$ and a number $M$ such that 
if $D^{\prime}=\left\{x_{i}\right\}_{i=0}^{n}$ is a refinement of $D$ and $0<p<q \leqq n$, then $\left|\Pi_{i=p}^{q}\left(1+G_{i}\right)^{-1}\right|<M[13$, Lemma 1].

LEMma 1.5. If $G$ is a function from $R \times R$ to $R$ such that $\int_{a}^{b} G^{2}=0$ and $\int_{a}^{b} G$ exists, then there is a subdivision $D$ of $[a, b]$ and a number $M$ such that if $D^{\prime}=\left\{x_{i}\right\}_{i=0}^{n}$ is a refinement of $D$ and $0<p<q \leqq n$, then $\left|\prod_{i=p}^{q}\left(1+G_{i}\right)^{-1}\right|<M$.

Indication of proof. Since $\int_{a}^{b} G^{2}=0$ and $\int_{a}^{b} G$ exists, then from Lemma 1.2, ${ }_{a} \Pi^{b}(1+G)$ exists and is not zero. ${ }_{a}$ Hence, since $\int_{a}^{b} G^{2}=0$ implies that $\left|G_{i}\right|<1$ for any refinement $D^{\prime}=\left\{x_{i}\right\}_{i=0}^{n}$ of an appropriate subdivision of $[a, b]$, then Lemma 1.5 follows from Lemma 1.4.

LEMMA 1.6. If $G$ is a function from $R \times R$ to $R$ such that $\int_{a}^{b} G$ exists and for each $x<y, H(x, y)=\left|\int_{x}^{y} G-G(x, y)\right|$, then $\int_{a}^{b} H$ exists and is 0 .

This lemma is due to A. Kolmogoroff [14]. For related results the reader is referred to W. D. L. Appling [1, Theorem 1.2] and B. W. Helton [4, Theorem 4.1].

LEMma 1.7. If $G$ is a function from $R \times R$ to $R$ such that $G$ is bounded on $[a, b],{ }_{a} \Pi^{b}(1+G)$ exists and is not zero, and $H$ is a function from $R \times R$ to $R$ such that for each $a \leqq x<y \leqq b, H(x, y)=$ $\left|1+G(x, y)-{ }_{x} \Pi^{y}(1+G)\right|$, then $\int_{a}^{b} H$ exists and is 0 [6, Lemma 1.4].

LEMma 1.8. If each of $H$ and $G$ is a function from $R \times R$ to $R$ such that ${ }_{a} \Pi^{b}(1+H)$ exists and ${ }_{a} \Pi^{b}(1+G)$ exists and neither is zero, then ${ }_{a} \Pi^{b}(1+H)(1+G)$ exists and is not zero.

Proof. The proof of this lemma is straightforward and we omit it.

LeMma 1.9. If $G$ is a function from $R \times R$ to $R, \int_{a}^{b} G$ exists, and $G$ is bounded on $[a, b]$ then there is a subdivision $\stackrel{a}{D}$ of $[a, b]$ and a number $M$ such that if $D^{\prime}=\left\{x_{i}\right\}_{i=0}^{n}$ is a refinement of $D$ and $0<p<q \leqq n$, then $\left|\sum_{i=p}^{q} G_{i}\right|<M$.

Proof. This lemma follows from Lemma 1.6.

The following algebraic identity is used frequently and it may be established by induction. 
LEMMA 1.10. If each of $\left\{a_{i}\right\}_{i=1}^{n}$ and $\left\{b_{i}\right\}_{i=1}^{n}$ is a sequence of numbers and $n>1$, then

$$
\prod_{i=1}^{n} a_{i}-\prod_{i=1}^{n} b_{i}=\sum_{i=1}^{n}\left(\prod_{j=1}^{i-1} b_{j}\right)\left(a_{i}-b_{i}\right)\left(\prod_{j=i+1}^{n} a_{j}\right) .
$$

THEOREM 1. Suppose each of $G, H$, and $J$ is a function from $R \times R$ to $R$ such that $J$ is of bounded variation on $[a, b], \int_{a}^{b} G^{2}=$ $\int_{a}^{b} H^{2}=0, \int_{a}^{b} J$ exists, for each $a \leqq x<y \leqq b$, each of ${ }_{x} \Pi^{y}(1+G)$ and ${ }_{x} \Pi^{y}(1+H)$ exists and neither is zero, $(R R) \int_{a}^{b} J\left[{ }_{y} \Pi^{t}(1+G)\right]\left[\Pi_{t} \Pi^{y}(1+H)\right]$ exists, and for each $a \leqq x<y \leqq b$,

$$
K(x, y)=\left|J(x, y)-(R R) \int_{x}^{y} J\left[{ }_{y} \Pi^{t}(1+G)\right]\left[{ }_{t} \Pi^{y}(1+H)\right]\right| .
$$

Then, $\int_{a}^{b} K$ exists and is 0 .

Proof. Let $\varepsilon>0$. Since $\int_{a}^{b} J$ exists and $J$ is of bounded variation on $[a, b]$, then, from Lemma 1.6 , there is a subdivision $D_{1}$ of $[a, b]$ and a number $M_{1}$ such that if $D^{\prime}=\left\{x_{i}\right\}_{i=0}^{n}$ is a refinement of $D_{1}$, then

$$
\sum_{D^{\prime}}\left|J_{i}\right|<M
$$

and

$$
\sum_{D^{\prime}}\left|\int_{x_{i-1}}^{x_{i}} J-J_{i}\right|<\frac{\varepsilon}{4}
$$

Since $\int_{a}^{b} G^{2}=\int_{a}^{b} H^{2}=0$ and each of ${ }_{a} \Pi^{b}(1+G)$ and ${ }_{a} \Pi^{b}(1+H)$ exists and neither is zero then, from Lemma 1.2, $\int_{a}^{b} G$ and $\int_{a}^{b} H$ each exists, and there is a subdivision $D_{2}$ of $[a, b]$ and a number $M_{2}$ such that if $D^{\prime}=\left\{x_{i}\right\}_{i=0}^{n}$ is a refinement of $D_{2}$, then

$$
\begin{gathered}
\left|\exp \int_{x_{i-1}}^{x_{i}} G\right|<M_{2}, \\
\left|\exp \int_{x_{i-1}}^{x_{i}} H\right|<M_{2}, \\
\left|G\left(x_{i-1}, x_{i}\right)\right|<\frac{1}{6} \ln \left(1+\frac{\varepsilon}{4 M_{1} M_{2}^{3}}\right),
\end{gathered}
$$

and

$$
\left|H\left(x_{i-1}, x_{i}\right)\right|<\frac{1}{6} \ln \left(1+\frac{\varepsilon}{4 M_{1} M_{2}^{3}}\right) .
$$

Again, from Lemma 1.6 there is a subdivision $D_{3}$ of $[a, b]$ such that 
if $D^{\prime}=\left\{x_{i}\right\}_{i=0}^{n}$ is a refinement of $D_{3}$, then

$$
\sum_{D^{\prime}}\left|\left(G_{i}-H_{i}\right)-\int_{x_{i-1}}^{x_{i}}(G-H)\right|<\frac{1}{6} \ln \left(1+\frac{\varepsilon}{M_{1} M_{2}^{3}}\right) .
$$

Let $D=D_{1}+D_{2}+D_{3}$ and $D^{\prime}=\left\{x_{i}\right\}_{i=0}^{n}$ be a refinement of $D$. Since, for $0<i \leqq n, \quad(R R) \int_{x_{i-1}}^{x_{i}} J\left[x_{i} \Pi^{t}(1+G)\right]\left[\Pi_{t} \Pi^{x_{i}}(1+H)\right]$ exists, then there is a subdivision $D_{i}=\left\{t_{j}\right\}_{j=0}^{k_{i}}$ of $\left[x_{i-1}, x_{i}\right]$ such that

$$
\begin{aligned}
& \mid \sum_{D_{i}}\left[J\left(t_{j-1}, t_{j}\right)\right]\left[_{x_{i}} \Pi^{t_{j}}(1+G)\right]\left[t_{j} \Pi^{x_{i}}(1+H)\right] \\
& \quad-\int_{x_{i-1}}^{x_{i}} J\left[_{x_{i}} \Pi^{t}(1+G)\right]\left[\Pi_{t} \Pi^{x_{i}}(1+H)\right] \mid<\frac{\varepsilon}{4 \cdot 2^{i}} .
\end{aligned}
$$

Therefore, for $x_{i-1} \leqq t_{j} \leqq x_{i}$,

$$
\begin{aligned}
\mid \int_{x_{i-1}}^{x_{i}} & (G-H)-\int_{x_{i-1}}^{t_{j}}(G-H) \mid \\
\leqq & \left|\int_{x_{i-1}}^{x_{i}}(G-H)-\left(G_{i}-H_{i}\right)\right|+\left|G\left(x_{i-1}, t_{j}\right)-H\left(x_{i-1}, t_{j}\right)-\int_{x_{i-1}}^{t_{j}}(G-H)\right| \\
& \quad+\left|G\left(x_{i-1}, t_{j}\right)\right|+\left|H\left(x_{i-1}, t_{j}\right)\right|+\left|G\left(x_{i-1}, x_{i}\right)\right|+\left|H\left(x_{i-1}, x_{i}\right)\right| \\
& <6\left[\frac{1}{6} \ln \left(1+\frac{\varepsilon}{4 M_{1} M_{2}^{3}}\right)\right] \\
= & \ln \left(1+\frac{\varepsilon}{4 M_{1} M_{2}^{3}}\right) .
\end{aligned}
$$

Hence, from (9) it follows that

$$
\left|\exp \int_{x_{i-1}}^{x_{i}}(G-H)-\exp \int_{x_{i-1}}^{t_{j}}(G-H)\right|<\frac{\varepsilon}{4 M_{2}^{2} M_{1}} .
$$

Then,

$$
\begin{aligned}
\sum_{D^{\prime}}\left|K_{i}\right|= & \sum_{D^{\prime}}\left|J_{i}-\int_{x_{i-1}}^{x_{i}} J\left[_{x_{i}} \Pi^{t}(1+G)\right]\left[\Pi_{t} \Pi^{x_{i}}(1+H)\right]\right| \\
\leqq & \sum_{D^{\prime}}\left|J_{i}-\int_{x_{i-1}}^{x_{i}} J\right| \\
& +\sum_{D^{\prime}}\left|\int_{x_{i-1}}^{x_{i}} J-\sum_{D_{i}} J\left(t_{j-1}, t_{j}\right)\right| \\
& +\sum_{D^{\prime}}\left|\sum_{D_{i}} J\left(t_{j-1}, t_{j}\right)-\sum_{D_{i}}\left[J\left(t_{j-1}, t_{j}\right)\right]\left[_{x_{i}} \Pi^{t_{j}}(1+G)\right]\left[_{t_{j}} \Pi^{x_{i}}(1+H)\right]\right| \\
& +\sum_{D^{\prime}} \mid \sum_{D_{i}}\left[J\left(t_{j-1}, t_{j}\right)\right]\left[x_{x_{i}} \Pi^{t_{j}}(1+G)\right]\left[t_{t_{j}} \Pi^{x_{i}}(1+H)\right] \\
& -\int_{x_{i-1}}^{x_{i}} J\left[\left[_{x_{i}} \Pi^{t}(1+G)\right]\left[\Pi_{t} \Pi^{x_{i}}(1+H)\right] \mid\right. \\
\leqq & \frac{\varepsilon}{4}+\sum_{D^{\prime}}\left|\sum_{D_{i}} \int_{t_{j-1}}^{t_{j}} J-\sum_{\nu_{i}} J\left(t_{j_{-1}}, t_{j}\right)\right|
\end{aligned}
$$




$$
\begin{aligned}
& +\sum_{D^{\prime}} \sum_{\nu_{i}}\left|J\left(t_{j-1}, t_{j}\right)\right| \cdot\left|1-{ }_{x_{i}} \Pi^{t_{j}}(1+G)_{t_{j}} \Pi^{x_{i}}(1+H)\right| \\
& +\sum_{D^{\prime}} \frac{\varepsilon}{4 \cdot 2^{i}} \\
\leqq & \sum_{D^{\prime}} \sum_{D_{i}}\left|\int_{t_{j-1}}^{t_{j}} J-J\left(t_{j-1}, t_{j}\right)\right| \\
& +\sum_{D^{\prime}} \sum_{D_{i}}\left|J\left(t_{j-1}, t_{j}\right)\right|\left|1-\left[\exp \int_{x_{i}}^{t_{j}} G\right]\left[\exp \int_{t j}^{x_{i}} H\right]\right| \\
& +\frac{\varepsilon}{2} \\
\leqq & \frac{\varepsilon}{4}+\sum_{D^{\prime}} \sum_{D_{i}}\left|J\left(t_{j-1}, t_{j}\right)\right| \cdot\left|\exp \int_{x_{-1}}^{x_{i}}(G-H)\right| \\
& \times\left|\exp \int_{x_{i-1}}^{x_{i}}(G-H)-\exp \int_{x_{i-1}}^{t_{j}}(G-H)\right| \\
& +\frac{\varepsilon}{2} \\
\leqq & M_{2}^{2} \sum_{D^{\prime}} \sum_{D_{i}}\left|J\left(t_{j-1}, t_{j}\right)\right| \frac{\varepsilon}{4 M_{1} M_{2}^{2}}+\frac{3 \varepsilon}{4} \\
< & M_{1} \cdot \frac{\varepsilon}{4 M_{1}}+\frac{3 \varepsilon}{4} \\
= & \varepsilon
\end{aligned}
$$

Hence, $\int_{a}^{b} K=0$.

We now state the main result of this paper.

THEOREM 2. Suppose each of $G$ and $H$ is a function from $R \times R$ to $R$ and each of $f$ and $h$ is a function from $R$ to $R$ such that $d h$ is of bounded variation on $[a, x]$ and $\int_{a}^{x} H^{2}=\int_{a}^{x} G^{2}=0$ for $x>a$.

The following two statements are equivalent:

(1) If $x>a$, then $f$ is bounded on $[a, x], \int_{a}^{x} H$ exists, $\int_{a}^{x} G$ exists, $(R L) \int_{a}^{x}(f G+f H)$ exists, and

$$
f(x)=h(x)+(R L) \int_{a}^{x}(f G+f H) ;
$$

(2) If $a \leqq p<q \leqq x$, then each of ${ }_{p} \Pi^{q}(1+H)$ and ${ }_{p} \Pi^{q}(1-G)^{-1}$ exists and neither is zero, $(R) \int_{a}^{x}\left[\Pi_{t} \Pi^{x}(1+H)(1+G)\right]\left[(1-G)^{-1}\right] d h$ exists, and

$$
\begin{aligned}
f(x)= & f(a){ }_{a} \Pi^{x}(1+H)(1-G)^{-1} \\
& +(R) \int_{a}^{x}\left[\Pi_{t} \Pi^{x}(1+H)(1+G)\right]\left[(1-G)^{-1}\right] d h .
\end{aligned}
$$

Proof. $1 \Rightarrow 2$. Let $a \leqq p<q \leqq x$. Since $\int_{a}^{x} H^{2}=\int_{a}^{x} G^{2}=0$ and each of $\int_{a}^{x} H$ and $\int_{a}^{x} G$ exists, then, from Lemma 1.2, ${ }_{p} \Pi^{q}(1+H)$ and 
${ }_{p} \Pi^{q}(1-G)$ exist and neither is 0 , and hence $\left[{ }_{p} \Pi^{q}(1-G)\right]^{-1}=$ ${ }_{p} \Pi^{q}(1-G)^{-1}$ exists. Since $\int_{a}^{x} G^{2}=0$, then, from Lemma $1.3,(1-G)^{-1}$ is bounded on $[a, x]$ and since $d h$ has bounded variation on $[a, x]$, then $(1-G)^{-1} d h$ has bounded variation on $[a, x]$. Let $\varepsilon>0$. Since $d h$ is of bounded variation on $[a, x]$ then there is a number $M_{1}$ and a subdivision $D_{1}$ of $[a, x]$ such that if $D^{\prime}=\left\{x_{i}\right\}_{i=0}^{n}$ is a refinement of $D_{1}$, then $\sum_{D^{\prime}}\left|d h_{i}\right|<M_{1}$. From, Lemma 1.5 , there is a number $M_{2}$ and a subdivision $D_{2}$ of $[a, x]$ such that if $D^{\prime}=\left\{x_{i}\right\}_{i=0}^{n}$ is a refinement of $D_{2}$ and $0<p<q \leqq n$, then $\left|\prod_{i=p}^{q}(1-G)^{-1}\right|<M_{2}$ Since $\int_{a}^{x} H$ exists then, from Lemma 1.9 , there is a subdivision $D_{3}$ of $[a, x]$ and a number $M_{3}$ such that if $D^{\prime}=\left\{x_{i}\right\}_{i=0}^{n}$ is a refinement of $D_{3}$ and $0<p<q \leqq n$, then $\left|\sum_{i=p}^{q} H_{i}\right|<M_{3}$. Since $(R L) \int_{a}^{b}(f G+f H)$ exists then there is a subdivision $D_{4}$ of $[a, x]$ such that if $D^{\prime}=\left\{x_{i}\right\}_{i=0}^{n}$ is a refinement of $D_{4}$, then

$$
\sum_{D^{\prime}}\left|(R L) \int_{x_{i-1}}^{x_{i}}(f G+f H)-\left(f_{i} G_{i}+f_{i-1} H_{i}\right)\right|<\frac{\varepsilon}{3 M_{2}^{2} \exp M_{3}} .
$$

Also, there exists a subdivision $D_{5}$ of $[a, x]$ such that if $D^{\prime}=\left\{x_{i}\right\}_{i=0}^{n}$ is a refinement of $D_{5}$ then

$$
\left|{ }_{a} \Pi^{x}(1+H){ }_{a} \prod_{D^{\prime}}{ }^{x}(1-G)^{-1}-\prod_{E^{\prime}}\left(1+H_{i}\right)\left(1-G_{i}\right)^{-1}\right|<\frac{\varepsilon}{3(|f(a)|+1)}
$$

and

$$
\begin{aligned}
& \text { if } 0<p<q \leqq n, \\
& \qquad\left|\prod_{j=p}^{q}\left(1+H_{j}\right)\left(1-G_{j}\right)^{-1}-{ }_{x_{p}} \Pi^{x_{q}}(1+H)(1-G)^{-1}\right|<\frac{\varepsilon}{3 M_{1} M_{2}} .
\end{aligned}
$$

Let $D=D_{1}+D_{2}+D_{3}+D_{4}+D_{5}$ and $D^{\prime}=\left\{x_{i}\right\}_{i=0}^{n}$ be a refinement of $D$, then, from the iterative technique of $\mathrm{B}$. W. Helton [4, page 311], we have that

$$
\begin{aligned}
f(x)= & f\left(x_{n}\right) \\
= & f_{0}\left[\prod_{i=1}^{n}\left(1+H_{i}\right)\left(1-G_{i}\right)^{-1}\right] \\
& +\prod_{i=1}^{n}\left[\prod_{j=i+1}^{n}\left(1+H_{j}\right)\left(1-G_{j}\right)^{-1}\right]\left(1-G_{i}\right)^{-1} d h_{i} \\
& +\prod_{i=1}^{n}\left(1-G_{i}\right)^{-1}\left[\prod_{j=i+1}^{n}\left(1+H_{j}\right)\left(1-G_{j}\right)^{-1}\right] \\
& \times\left[(R L) \int_{x_{i-1}}^{x_{i}}(f G+f H)-\left(f_{i} G_{i}+f_{i-1} H_{i}\right)\right] .
\end{aligned}
$$

Hence, 


$$
\begin{aligned}
\mid f(x)- & f(a)_{a} \Pi^{x}(1+H)_{a} \Pi^{x}(1-G)^{-1}-\sum_{D^{\prime}}\left[x_{i} \Pi^{x}(1+H)(1+G)\right]\left[\left(1-G_{i}^{-1}\right)\right] d h_{i} \mid \\
\leqq & \left|f(a)\left[\prod_{D^{\prime}}\left(1+H_{i}\right)\right]\left[\left(1-G_{i}\right)^{-1}\right]-f(a)_{a} \Pi^{x}(1+H)_{a} \Pi^{x}(1-G)^{-1}\right| \\
& +\mid \sum_{D^{\prime}}\left[\prod_{j=i+1}^{n}\left(1+H_{i}\right)\right]\left[\prod_{j=i+1}^{n}\left(1-G_{i}\right)^{-1}\right]\left[\left(1-G_{i}\right)^{-1}\right] d h_{i} \\
& -\sum_{D^{\prime}}\left[x_{i} \Pi^{x}(1+H)(1+G)^{-1}\right]\left[\left(1-G_{i}\right)^{-1}\right] d h_{i} \mid \\
& +\sum_{D^{\prime}}\left|\left[\prod_{j=i+1}^{n}\left(1+H_{j}\right)\right]\left[\prod_{j=i+1}^{n}\left(1-G_{j}\right)^{-1}\right]\left[\left(1-G_{i}\right)^{-1}\right]\right| \\
& \times\left|(R L) \int_{x_{i-1}}^{x_{i}}(f G+f H)-\left(f_{i} G_{i}+f_{i-1} H_{i}\right)\right| \\
\leqq & |f(a)| \cdot \frac{\varepsilon}{3[|f(a)|+1]} \\
& +\sum_{D^{\prime}}\left|\left(1-G_{i}\right)^{-1}\right| \cdot\left|d h_{i}\right| \cdot \mid \prod_{j=i+1}^{n}\left(1+H_{i}\right)\left(1-G_{i}\right)^{-1} \\
& -\left[x_{i} \Pi^{x}(1+H)(1-G)^{-1}\right] \mid \\
& +\sum_{D^{\prime}}\left|\exp \sum_{j=i+1}^{n} H_{j}\right| \cdot M_{2} \cdot M_{2} \cdot\left|(R L) \int_{x_{i-1}}^{x_{i}}(f G+f H)-\left(f_{i} G_{i}+f_{i-1} H_{i}\right)\right| \\
< & \frac{\varepsilon}{3}+M_{2} \cdot \frac{\varepsilon}{3 M_{1} M_{2}} \sum_{D^{\prime}}\left|d h_{i}\right|+M_{2}^{2} \exp M_{3}\left[\frac{\varepsilon}{\left.3 M_{2}^{2} \exp M_{3}\right]}\right. \\
< & \frac{\varepsilon}{3}+\frac{\varepsilon}{3 M_{1}} \cdot M_{1}+\frac{\varepsilon}{3} \\
= & \varepsilon
\end{aligned}
$$

Hence, $(R) \int_{a}^{x}\left[\Pi_{t} \Pi^{x}(1+H)(1+G)\right]\left[(1-G)^{-1}\right] d h$ exists and $f(x)=f(a)_{a} \Pi^{x}(1+H)(1-G)^{-1}+(R) \int_{a}^{x}\left[\Pi_{t} \Pi^{x}(1+H)(1+G)\right]\left[(1-G)^{-1}\right] d h$.

$2 \Rightarrow 1$. Suppose $x>a$ and $\varepsilon>0$. Since each of ${ }_{a} \Pi^{x}(1+H)$ and ${ }_{a} \Pi^{x}(1-G)^{-1}$ exists and neither is 0 , then, from Lemma 1.2, $\int_{a}^{x} H$ exists, $\int_{a}^{x} G$ exists, and from Lemma 1.1, each of $H$ and $G$ is bounded on $[a, x]$. From Lemma 1.5, $(1-G)^{-1}$ is bounded on $[a, x]$ and since $d h$ is of bounded variation $[a, x]$, then $d h$ is bounded on $[a, x]$. Therefore, it follows from the boundedness of the functions involved that $f$ is bounded on $[a, x]$. Hence, there is a number $M$ and a subdivision $D_{1}$ of $[a, x]$ such that if $D^{\prime}=\left\{x_{i}\right\}_{i=0}^{n}$ is a refinement of $D_{1}$ and $0<i \leqq n$, then (1) $\left|f_{i-1}\right|<M$ and (2) $\left|1-G_{i}\right|<M$.

Since $(1-G)^{-1}$ is bounded on $[a, x]$ and hence $\left[(1-G)^{-1}\right] d h$ is of bounded variation on $[a, x]$, then from Theorem 1 , there is a subdivision $D_{2}$ of $[a, x]$ such that if $D^{\prime}=\left\{x_{i}\right\}_{i=0}^{n}$ is a refinement of $D_{2}$, then 


$$
\sum_{D^{\prime}}\left|(R) \int_{x_{i-1}}^{x_{i}}\left[\Pi_{t} \Pi^{x_{i}}(1+H)(1-G)^{-1}\right]\left[(1-G)^{-1}\right] d h-\left[\left(1-G_{i}\right)^{-1}\right] d h_{i}\right|<\frac{\varepsilon}{3 M} .
$$

Furthermore, since ${ }_{a} \Pi^{x}(1+H)(1-G)^{-1}$ exists and is not zero, then from Lemma 1.7, there is a subdivision $D_{3}$ of $[a, x]$ such that if $D^{\prime}=\left\{x_{i}\right\}_{i=0}^{n}$ is a refinement of $D_{3}$, then

$$
\left.\sum_{D^{\prime}}\right|_{x_{i-1}} \Pi^{x_{i}}(1+H)(1-G)^{-1}-\left(1+H_{i}\right)\left(1-G_{i}\right)^{-1} \mid<\frac{\varepsilon}{3 M^{2}} .
$$

If $D=D_{1}+D_{2}+D_{3}$ and $D^{\prime}=\left\{x_{i}\right\}_{i=0}^{n}$ is a refinement of $D$, then, again using the iterative technique employed by B. W. Helton in [4, page 312], we have, for $0<i \leqq n$,

$$
\begin{aligned}
f_{i}= & f_{i-1 x_{i-1}} \Pi^{x_{i}}(1+H)(1-G)^{-1}+(R) \int_{x_{i-1}}^{x_{i}}\left[\Pi_{t} \Pi^{x_{i}}(1+H)(1-G)^{-1}\right]\left[(1-G)^{-1}\right] d h \\
= & f_{i-1}\left(1+H_{i}\right)\left(1-G_{i}\right)^{-1}+f_{i-1}\left[x_{i-1} \Pi^{x_{i}}(1+H)(1-G)^{-1}-\left(1+H_{t}\right)\left(1-G_{i}\right)^{-1}\right] \\
& +d h_{i}\left(1-G_{i}\right)^{-1} \\
& +(R) \int_{x_{i-1}}^{x_{i}}\left[\Pi^{x_{i}}(1+H)(1-G)^{-1}\right](1-G)^{-1} d h-d h_{i}\left(1-G_{i}\right)^{-1} .
\end{aligned}
$$

By multiplying both sides of the preceding equation by $\left(1-G_{i}\right)$ and then rearranging terms, we have

$$
\begin{aligned}
f_{i}-f_{i-1}= & f_{i} G_{i}+f_{i-1} H_{i}+d h_{i} \\
& +f_{i-1}\left[x_{i-1} \Pi^{x_{i}}(1+H)(1-G)^{-1}-\left(1+H_{i}\right)\left(1-G_{i}\right)^{-1}\right]\left[1-G_{i}\right] \\
& +\left(1-G_{i}\right)\left[(R) \int_{x_{i-1}}^{x_{i}}\left[\Pi_{t}^{x_{i}}(1+H)(1-G)^{-1}\right]\left[(1-G)^{-1}\right] d h\right. \\
& \left.-\left[(1-G)^{-1}\right] d h_{i}\right] .
\end{aligned}
$$

Therefore,

$$
\begin{aligned}
\mid f(x) & -h(x)-\sum_{D^{\prime}}\left(f_{i} G_{i}+f_{i-1} H_{i}\right) \mid \\
= & \left|f(x)-f(a)+h(a)-h(x)-\sum_{D^{\prime}}\left(f_{i} G_{i}+f_{i-1} H_{i}\right)\right| \\
= & \left|\sum_{D^{\prime}}\left(f_{i}-f_{i-1}\right)-\sum_{D^{\prime}} d h_{i}-\sum_{D^{\prime}}\left(f_{i} G_{i}+f_{i-1} H_{i}\right)\right| \\
\leqq & \left|\sum_{D^{\prime}} d h_{i}-\sum_{D^{\prime}} d h_{i}\right| \\
& +\left.\sum_{D^{\prime}}\left|f_{i-1}\right| \cdot\left|1-G_{i}\right| \cdot\right|_{x_{i-1}} \Pi^{x_{i}}(1+H)(1-G)^{-1}-\left(1+H_{i}\right)\left(1-G_{i}\right)^{-1} \mid \\
& +\sum_{D^{\prime}}\left|1-G_{i}\right| \cdot \mid(R) \int_{x_{i-1}}^{x_{i}}\left[\Pi^{x_{i}}(1+H)(1-G)^{-1}\right]\left[(1-G)^{-1}\right] d h \\
& -\left(1-G_{i}\right)^{-1} d h_{i} \mid \\
< & 0+\left.M^{2} \sum_{D^{\prime}}\right|_{x_{i-1}} \Pi^{x_{i}}(1+H)(1-G)^{-1}-\left(1+H_{i}\right)\left(1-G_{i}\right)^{-1} \mid
\end{aligned}
$$




$$
\begin{aligned}
& \quad+M \sum_{D^{\prime}}\left|(R) \int_{x_{i-1}}^{x_{i}}\left[{ }_{t} \Pi^{x_{i}}(1+H)(1-G)^{-1}\right]\left[(1-G)^{-1}\right] d h-(1-G)^{-1} d h_{i}\right| \\
& <M^{2} \frac{\varepsilon}{3 M^{2}}+M \frac{\varepsilon}{3 M} \\
& <\varepsilon .
\end{aligned}
$$

Hence, $(R L) \int_{a}^{x}(f G+f H)$ exists and $f(x)=h(x)+(R L) \int_{a}^{x}(f G+f H)$.

\section{REFERENCES}

1. W. D. L. Appling, Interval functions and real Hilbert spaces, Rend. Circ. Mat. Plermo, 2 (1962), 154-154.

2. J. A. Chatfield, Three Volterra integral equations, Tex. J. Sci., 27 (1976), 33-38.

3. W. P. Davis and J. A. Chatfield, Concerning product integrals and exponentials, Proc. Amer. Math. Soc., 25 (1970), 743-747.

4. B. W. Helton, Integral equations and product integrals, Pacific J. Math., 16 (1966), 297-322.

5. - A product integral solution of a Riccati equation, Pacific J. Math., 56 (1975), 113-130.

6. J. C. Helton, Existence of sum and product integrals, Trans. Amer. Math. Soc., 182 (1973), 165-174.

7. - Product integrals and inverses in normed rings, Pacific J. Math., 51 (1974), 155-166.

8. - Solution of integral equations by product integration, Proc. Amer. Math. Soc., 49 (1975), 401-406.

9. - Product integrals and the solution of integral equations, Pacific J. Math., 58 (1975), 87-103.

10. — Product integrals, bounds, and inverses, Tex. J. Sci., 26 (1975), 11-18.

11. - Bounds for products of interval functions, Pacific J. Math., 49 (1973), $377-389$.

12. - Some interdependencies of sum and product integrals, Proc. Amer. Math. Soc., 37 (1973), 201-206.

13. - Product integrals and exponentials in commutative Banach algebras, Proc. Amer. Math. Soc., 39 (1973), 155-162.

14. A. Kolmogoroff, Untersuchungen uber den Integralbegriff, Math. Ann., 103 (1930), 654-696.

15. Davis Lowell Lovelady, Perturbations of solutions of Stieltjes integral equations, Trans. Amer. Math. Soc., 155 (1971), 175-187.

16. Bounded solutions of Stieltjes integral equations, Proc. Amer. Math. Soc., 28 (1971), 127-133.

17. J. S. MacNerney, Integral equations and semigroups, Illinois J. Math., 7 (1963), 148-173.

18. J. W. Neuberger, Continuous products and nonlinear integral equations, Pacific J. Math., 8 (1958), 529-549.

Received July 16, 1976.

Southwest Texas State University

SAN MARCOS, TX 78666 



\section{PACIFIC JOURNAL OF MATHEMATICS}

\section{EDITORS}

RICHARD ARENS (Managing Editor)

University of California

Los Angeles, California 90024

C. W. CURTIS

University of Oregon

Eugene, OR 97403

C. C. MOORE

University of California

Berkeley, CA 94720
J. DugundjI

Department of Mathematics University of Southern Californı Los Angeles, California 90007

R. Finn and J. Milgram Stanford University Stanford, California 94305

ASSOCIATE EDITORS

E. F. BECKENBACH

B. H. NeUmanN

F. WOLF

K. YoSHIDA

\section{SUPPORTING INSTITUTIONS}

UNIVERSITY OF BRITISH COLUMBIA CALIFORNIA INSTITUTE OF TECHNOLOGY

UNIVERSITY OF CALIFORNIA

MONTANA STATE UNIVERSITY

UNIVERSITY OF NEVADA, RENO

NEW MEXICO STATE UNIVERSITY

OREGON STATE UNIVERSITY

UNIVERSITY OF OREGON
UNIVERSITY OF SOUTHERN CALIFORNIA

STANFORD UNIVERSITY

UNIVERSITY OF HAWAII

UNIVERSITY OF TOKYO

UNIVERSITY OF UTAH

WASHINGTON STATE UNIVERSITY

UNIVERSITY OF WASHINGTON 


\section{Pacific Journal of Mathematics}

\section{Vol. 80, No. $1 \quad$ September, 1979}

Jeroen Bruijning and Jun-iti Nagata, A characterization of covering dimension by

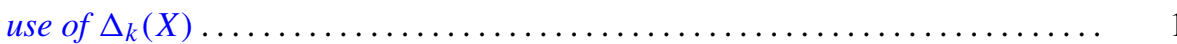

John J. Buoni and Albert Jonathan Klein, On the generalized Calkin algebra ...... 9

Thomas Ashland Chapman, Homotopy conditions which detect simple homotopy

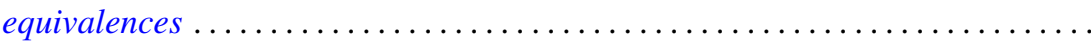

John Albert Chatfield, Solution for an integral equation with continuous interval

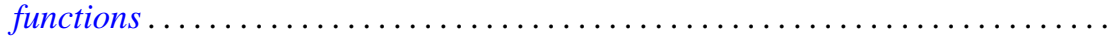

Ajit Kaur Chilana and Ajay Kumar, Spectral synthesis in Segal algebras on

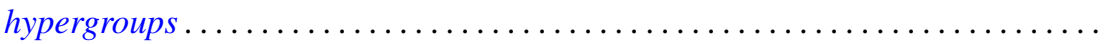

Lung O. Chung, Jiang Luh and Anthony N. Richoux, Derivations and

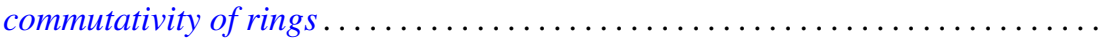

Michael George Cowling and Paul Rodway, Restrictions of certain function spaces to closed subgroups of locally compact groups .....................

David Dixon, The fundamental divisor of normal double points of surfaces........

Hans Georg Feichtinger, Colin C. Graham and Eric Howard Lakien,

Nonfactorization in commutative, weakly selfadjoint Banach algebras . . . . . . .

Michael Freedman, Cancelling 1-handles and some topological imbeddings ....... .

Frank E., III Gerth, The Iwasawa invariant $\mu$ for quadratic fields . . . . . . . . . . . . . .

Maurice Gilmore, Three-dimensional open books constructed from the identity

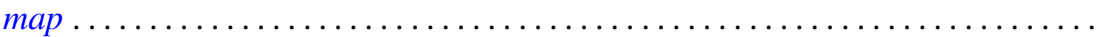

Stanley P. Gudder, A Radon-Nikodým theorem for $*$-algebras .

Peter Wamer Harley, III and George Frank McNulty, When is a point Borel? .

Charles Henry Heiberg, Fourier series with bounded convolution powers . .

Rebecca A. Herb, Characters of averaged discrete series on semisimple real Lie

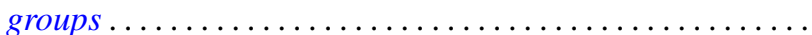

Hideo Imai, On singular indices of rotation free densities . .

Sushil Jajodia, On 2-dimensional CW-complexes with a single 2-cell . . .

Herbert Meyer Kamowitz, Compact operators of the form $u C_{\varphi}$

Matthew Liu and Billy E. Rhoades, Some properties of the Chebyshev method...

213

George Edgar Parker, Semigroups of continuous transformations and generating

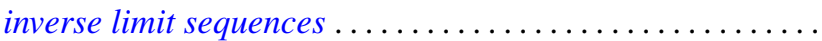

Samuel Murray Rankin, III, Oscillation results for a nonhomogeneous

equation ...

Martin Scharlemann, Transverse Whitehead triangulations ...

Gary Joseph Sherman, A lower bound for the number of conjugacy classes in a

finite nilpotent group

Richard Arthur Shoop, The Lebesgue constants for $\left(f, d_{n}\right)$-summability .

Stuart Jay Sidney, Functions which operate on the real part of a uniform

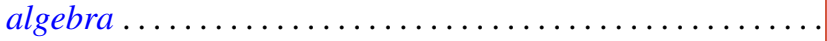

Tim Eden Traynor, The group-valued Lebesgue decomposition 